\title{
Review of Pre-Analytical Errors in Oral Glucose Tolerance Testing in a Tertiary Care Hospital
}

\author{
Rachita Nanda, ${ }^{1}$ Suprava Patel, ${ }^{1}$ Sibashish Sahoo, ${ }^{1}$ Eli Mohapatra ${ }^{1}$ \\ ${ }^{1}$ Department of Biochemistry, AlIMS, Raipur, Chhattisgarh, India.
}

\begin{abstract}
Background: The pre-pre-analytical and pre-analytical phases form a major chunk of the errors in a laboratory. The process has taken into consideration a very common procedure which is the oral glucose tolerance test to identify the pre-pre-analytical errors. Quality indicators provide evidence of quality, support accountability and help in the decision making of laboratory personnel. The aim of this research is to evaluate pre-analytical performance of the oral glucose tolerance test procedure.

Methods: An observational study that was conducted overa period of three months, in the phlebotomy and accessioning unit of our laboratory using questionnaire that examined the pre-pre-analytical errors through a scoring system. The pre-analytical phase was analyzed for each sample collected as per seven quality indicators.

Results: About 25\% of the population gave wrong answer with regard to the question that tested the knowledge of patient preparation. The appropriateness of test result QI-1 had the most error. Although QI-5 for sample collection had a low error rate, it is a very important indicator as any wrongly collected sample can alter the test result.

Conclusions: Evaluating the pre-analytical and pre-pre-analytical phase is essential and must be conducted routinely on a yearly basis to identify errors and take corrective action and to facilitate their gradual introduction into routine practice.

Keywords: Errors; pre-analytical; quality indicators.
\end{abstract}

\section{INTRODUCTION}

The pre-analytical phase represents an important stage in laboratory medicine and remains the most error prone $(70 \%)$ part of the laboratory. ${ }^{1}$ However, errors do occur outside the laboratory walls in the 'prepre-analytical phase'. The ISO 15189 recognizes and calls for evaluating, monitoring and improving all the procedures and processes in the pre-analytical and 'prepre-analytical phase'. ${ }^{1}$ This phase starts from request by the clinician, patient's preparation, collection of primary sample, transportation to and within the laboratory and ending when the analytical procedure begins. A quality indicator(QI) 'objectively measures all critical care domains, and evidence associated with those domains, that can be implemented in a consistent and comparable manner across settings and over time'. ${ }^{2}$ Oral glucose tolerance test(OGTT) a routinely conducted test requires proper preparation of patient about the procedure as it affects the accuracy and reliability. ${ }^{3}$ We utilized a set of QI based on the 'Harmonization of preanalytical quality indicators' to identify pre-analytical errors. ${ }^{4}$

The aim of this research is to evaluate pre-analytical performance of the OGTT procedure with the help of questionnaire and quality indicators so as to introduce them into daily practice.

\section{METHODS}

This observational study was conducted in the phlebotomy and accessioning unit of our laboratory after obtaining ethical clearance from our institute's ethical committee. The questionnaire designed was modified based on a study by Bankovic et $\mathrm{al}^{3}$ which pertained to examine their level of knowledge about the OGTT procedure. All patients attending the laboratory for OGTT over a period of three months were recruited for the study.

Correspondence: Dr. Rachita Nanda, Department of Biochemistry, Gate No -5, AlIMS, GE Road, Tatibandh, Chhattisgarh, Raipur, India. Email: dr.rachitananda@gmail.com, Phone: +918518881763 . 
The guidelines and information details about preparation of patients for OGTT in our laboratory follows standard information and is known to doctors and laboratory staff.

257 patients, all female presented at the laboratory for the OGTT procedure and were recruited for the study. This phase was evaluated by the questionnaire which was filled up while patients were in the sitting area prior to the procedure. Twenty two participants could not be included in the study as their questionnaire was incomplete. A total of 233 patients participated of which 95 were pregnant and rest 138 were non-pregnant who were placed in Group I and Group II respectively. The level of knowledge about preparation and about the procedure itself were calculated from correct answers to four questions (Table 1). Each correct answer was given one point, with 0 or $1=$ low score, $2=$ moderate score, $3=$ adequate score and $4=$ high score. The relevancy of these questions lies in their association to the understanding of the whole OGTT procedure.

The pre-analytical phase was analyzed for each sample collected as per the seven quality indicators (Table 2$)^{4}$. Each component of the quality indicator is assigned a priority score. The basis for identifying a priority scale for the $\mathrm{Ql}$ is to aid their ongoing introduction into regular practice by starting with a 'mandatory' (score 1) and ending with a 'valuable'(score 4) QI score. This will therefore help in raising the standard of quality management in the clinical laboratory.

Based on the response by the patients and data collected from patient prescription records and observation of the samples, the data was presented in numbers and percentages. After testing for distribution of normality of the variables, significance of means was tested by Mann-Whitney test, Kruskal-Wallis test and Chi square tests. The statistical analyses were performed by using SPSS 20.

\section{RESULTS}

All patients who participated in the study were females, from the department of Obstetrics and Gynecology. About $95 \% \quad(n=221)$ of the study population received information regarding the procedure from the laboratory staff, while rest of the patients received from their treating physician as well as laboratory personnel (Fig 1).

With regard to the level of knowledge about the OGTT procedure $75.5 \%$ revealed high, $17.16 \%$ adequate, 6.43 $\%$ moderate and $0.85 \%$ low level of knowledge (Table 3 ). The question which was answered with maximum wrong answers was to test the knowledge regarding preparation
(1 of Table 1).The average score of Group I was 3.54 and that of Group II was 3.76 with a significance of $p=0.05$. About $54 \%$ of participants were graduates and above in their level of education. The level of education affected the score which was evident by higher average score (3.8) in graduates and above (Fig 2). This was more than the mean score of the study population.

Table 1. Questions to assess the knowledge of

the OGTT procedure to detect pre pre analytical errors.

\begin{tabular}{lll} 
SN & Question & Answer \\
\hline 1. & $\begin{array}{l}\text { Several days } \\
\text { before the } \\
\text { examination } \\
\text { it is }\end{array}$ & $\begin{array}{l}\text {-Eat and move as little as } \\
\text { possible }\end{array}$ \\
& -Eat as usual, strictly rest \\
to : & -Eat as usual, avoid strenuous \\
2. & $\begin{array}{l}\text { Is fasting } \\
\text { important } \\
\text { on the day } \\
\text { of procedure } \\
\text { ? }\end{array}$ & YES/NO \\
\end{tabular}

3. After taking -to move as much as possible glucose until collection of -not to eat, drink, smoke and blood what move isimportant? -there are no special demands

4. What is -Exactly at $2 \mathrm{hr}$ after taking the time of glucose collection of the -In between 1-2 hr after next blood taking glucose sample?

-It is not strictly specified

SCORE High level - 4, Adequate level -3, Moderate level -2, Low level -1

We assumed that participants who declared that they had a knowledge regarding the OGTT procedure must have a better score. The self declaration of knowledge by participants matched with their real knowledge as was evident from score $(p=0.000)$. There was no difference in score with regard to the level of knowledge among participants who had $(n=44)$ or had not done $(n=189)$ OGTT in the past $(p=0.920)$. Participants who were previously pregnant $(n=103)$ had a better score $(p=0.015)$ than those who were never pregnant $(n=130$ ). Although the average score of Group I was low than Group II, the level of knowledge also improved with duration of pregnancy in weeks ( $p=0.011$ ) (Table 4). 
Table 2. Quality indicators in the pre-analytical phase.

QI-1. Appropriateness of test request

- $\quad$ Request without clinical question (2)

- Inappropriate test with respect to clinical question (4)

\section{QI-2. Patient Identification}

- Request with erroneous patient identification (1)

- Request with erroneous patient identification detected before release of results (1)

- Request with erroneous patient identification detected after release of results (1)

\section{QI-3. Data entry of request}

- Unintelligible requests (3)

- Request without physician's identification or with erroneous identification of physician (2)

- Request with errors concerning test input (missing) (1)

- $\quad$ Request with errors concerning test input (added) (1)

- $\quad$ Request with errors concerning test input (misinterpreted) (1)

QI-4. Sample identification

- Improperly labeled sampled (1)

QI-5. Sample collection

- Samples collected at inappropriate time (1)

- Samples of wrong or inappropriate type (1)

- Samples collected in inappropriate containers (1)

- Samples with insufficient volume (1)

QI-6. Transport of samples

- $\quad$ Samples damaged in transport (1)

- Samples transported at inappropriate temperature conditions (1)

- Samples lost/not received (1)

QI-7 Suitability of samples

- Samples with inadequate sample-anticoagulant ratio (2)

- $\quad$ Samples hemolyzed(1)

- $\quad$ Samples clotted (1)

- $\quad$ Samples lipemic(1)
*Based on the Model of Quality Indicators (MQI) proposed by IFCC Working Group 'Laboratory Errors and Patient Safety'(IFCC WG-LEPS) for pre-analytical phase(4). Each question of the QI is assigned a priority score starting with; 'Mandatory'- score 1 'Important'- score 2 'Suggested'-score 3'Valuable'-score 4

Table 3. Score for the level of knowledge about

preparation and procedure.

$\begin{array}{lll}\text { Total } & \text { Group I } & \text { Group II }(n \\ \text { population } & (n=95) & =138) \text { Non }\end{array}$

$(\mathrm{n}=233) \quad$ Pregnant Pregnant

\begin{tabular}{llll}
$\begin{array}{l}\text { High } \\
\text { score }\end{array}$ & $\begin{array}{l}176 \\
(75.5 \%)\end{array}$ & $64(67.3 \%)$ & $112(81 \%)$ \\
$\begin{array}{l}\text { Adequate } \\
\text { score }\end{array}$ & $\begin{array}{l}40 \\
(17.16 \%)\end{array}$ & $19(20 \%)$ & $21(15.2 \%)$ \\
$\begin{array}{l}\text { Moderate } \\
\text { score }\end{array}$ & $15(6.43 \%)$ & $12(12.6 \%)$ & $3(2.17 \%)$ \\
$\begin{array}{l}\text { Low score } \\
\text { Average } \\
\text { Score }\end{array}$ & $2(0.85 \%)$ & $0(0 \%)$ & $2(1.44 \%)$ \\
\hline
\end{tabular}

Table 4. Comparison of scores in various subgroups.

\begin{tabular}{|c|c|c|}
\hline Comparison Groups & Score & p Value \\
\hline $\begin{array}{l}\text { Previous Exposure OGTT }(n=44) \\
\text { Vs } \\
\text { No prior exposure to OGTT } \\
(n=189)\end{array}$ & $\begin{array}{l}3.68 \mathrm{Vs} \\
3.67\end{array}$ & 0.920 \\
\hline $\begin{array}{l}\text { Previously Pregnant }(n=103) \text { Vs } \\
\text { Never Pregnant }(n=130)\end{array}$ & $\begin{array}{l}3.69 \mathrm{Vs} \\
3.50\end{array}$ & 0.015 \\
\hline $\begin{array}{l}1^{\text {st }} \text { Trimester }(n=49) \text { Vs } 2^{\text {nd }} \\
\text { Trimester }(n=29) \text { Vs } 3^{\text {rd }} \\
\text { Trimester }(n=17)\end{array}$ & $\begin{array}{l}3.48 \mathrm{Vs} \\
3.56 \mathrm{Vs} \\
3.62\end{array}$ & 0.011 \\
\hline
\end{tabular}

The identification of pre-analytical errors was performed with the help of quality indicators as shown in Table 5. A total of 89 samples (38.19\%) had error for the QI-1 which is appropriateness of test request. Request without clinical question with priority score 2 and inappropriate test with respect to clinical question with priority score of 4 were seen in $47.1 \%$ and $52.9 \%$ of the errant. For QI2 , for patient identification, with priority score of 1 , the number of requests with errors before release of results and after issuing of results was 8 and 4 respectively. With regard to QI-3, the commonest error observed was none or erroneous identification of the physician. There was one sample each which was inappropriate and collected in wrong tube for QI-5. While checking for suitability of sample(QI-7), we found that 11 samples were hemolyzed and 4 were clotted. There was no error in QI-4 and QI- 6. 


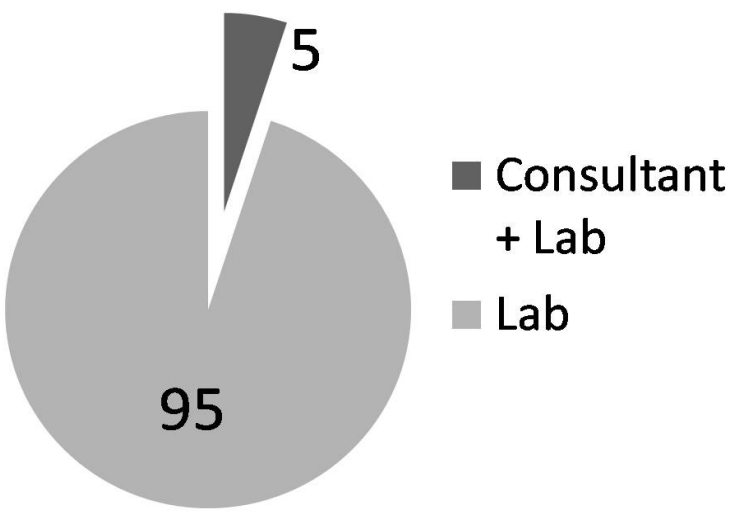

Figure 1. Source of information for OGTT.

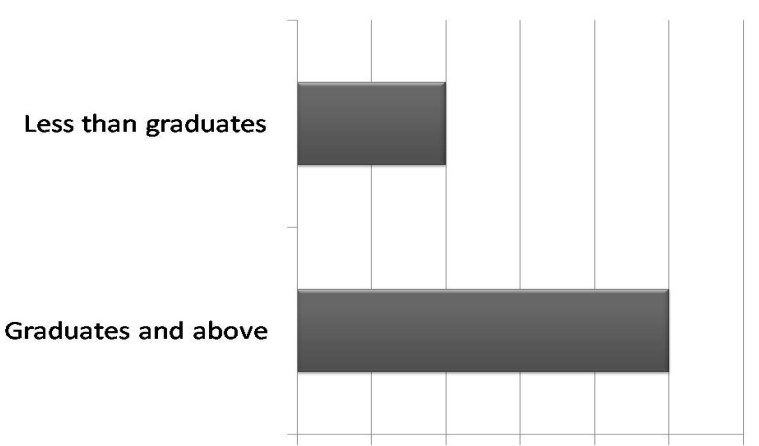

$\begin{array}{lllllll}3.3 & 3.4 & 3.5 & 3.6 & 3.7 & 3.8 & 3.9\end{array}$

Figure 2. Bar diagram to show score according to education status.

Table 5. Pre-analytical errors according to quality indicators.

$\%$ of errors

\begin{tabular}{llr}
\hline QI - 1 & Appropriateness of test request & $38 \%$ \\
\hline QI - 2 & Patient Identification & $5.1 \%$ \\
QI - 3 & Data entry of request & $12.8 \%$ \\
QI - 5 & Sample collection & $<0.8 \%$ \\
QI - 7 & Suitability of samples & $6.4 \%$ \\
\hline
\end{tabular}

\section{DISCUSSION}

Testing in a clinical laboratory consists of pre-pre analytical, pre-analytical, analytical and post analytical phases. With increased usage of automated machines, and computers the process involving the analytical phase and to a certain extent the post analytical phase have been simplified with an improvement in the error rate. However, the pre-analytical and pre-pre analytical phase still is an area of concern as the error rate can be even upto $70 \%$. Special attention has to be given to this phase to minimize the errors. This study scrutinized the pre-pre and pre-analytical performance for a common laboratory procedure i.e., OGTT.

OGTT is recommended for diagnosis of conditions like gestational diabetes mellitus, diagnosis of impaired glucose tolerance and also for population studies for epidemiological data.5,6 All the patients who had turned up at our laboratory for the testing were from the antenatal OPD to rule out GDM and were from the infertility clinic of Obstetric and Gynecology department. As is evident the patients (95\%) are dependent on the laboratory, who form the main source to obtain instructions regarding preparation for this procedure. To achieve quality sample and results proper patient preparation is essential as it can affect test results. One of the commonest preparation that is to be done is to be in a fasting state for blood sampling. For certain tests like OGTT mere fasting for $10-12$ hours is not enough. There are other specific instructions given to the patient that must be followed to ensure usefulness of performance of tests and correct interpretation of results.

Three fourth of the population were well prepared as they got correct answers to all questions, which was revealed by a high score. For the rest of the population, maximum error was found with regard to the preparation of the patient, and this could affect the consistency of the patient towards the technical procedure and thus the final test results. When comparing the two groups the non-pregnant group had a significant better average score than the pregnant group. The larger population of Group II were patients of secondary infertility, meaning they had previously been exposed to the OGTT procedure than Group I, where the patients included a larger number of women who were pregnant for the first time. Patients who were graduates and above had improved score indicating the receptiveness and understanding capability that is coupled to education. Previous pregnancy improved the score, but previously undertaken OGTT had no effect on the score. Even though the score was low in the pregnant group than in the non pregnant group, the score improved with duration of pregnancy demonstrating increased receptiveness.

The QI-1to assess the appropriateness of test ordered, had the maximum error. Ordering these tests could have unnecessarily added to the costs and potentially contribute to delayed and inappropriate clinical decision. Periodic feedback and reminders to clinicians may decrease the number of inappropriately ordered laboratory tests resulting in cost savings. ${ }^{7}$ With regard to $\mathrm{Q} I-2$, i.e., patient identification, such errors are undefended for. Such errors occur when proper patient identification errors are not followed and specimen tubes are incorrectly labelled. There were 12 samples who had this error. The test report form had the correct patient identification. However, while entering the details into the software these errors were introduces. 
These errors can be corrected by re-verifying each time an entry has been made. Also all technicians have to be trained and be made well versed with the phlebotomy and accessioning section of the laboratory.In order to improve the pre pre analytical and pre analytical phase the laboratory should provide physicians and other health care professionals a clear and understandable written instructions for patient preparation. One study by Singla et al. ${ }^{8}$ demonstrated that the custom of completion of request form was very poor.

Any request without physician's identification has an important priority score. This prevents communication of results to the physicians in case of critical values and thus delays appropriate medical intervention. A study by Nutt et al reported the details of physician was absent in $61.2 \%$ cases, and because of which $19.9 \%$ results which were critical. 9 Although there was one sample where the sample instead of being collected fluoride vacutainer was collected in a plain gel tube (golden top -SST), so serum was collected instead of plasma. However, in our lab as we get the samples we process it immediately and so in this case investigation was performed quickly. Nevertheless it is an indicator that retraining must be done and the guidelines regarding the choice of tubes must be available to the laboratory personnel at the phlebotomy and accessioning area. The suitability of the sample was hampered in 15 cases, due to hemolysis and clotting. Hemolysis lowers the glucose values, which could be due to early decomposition of hydrogen peroxide by hemoglobin as it has pseudoperoxidase activity. ${ }^{10}$ In severe hemolysis due to release of intracellular components, a dilutional effect is seen which could lower plasma glucose level. ${ }^{11}$ The most frequent pre analytical error in a clinical chemistry lab is hemolysis after centrifugation. ${ }^{12,13}$ However, the present day analytical systems have serum hemolysis index for detection of interference pre analytically for which laboratory staff must be made aware and take decisions.

Based on the above errors in the pre-pre-analytical and pre-analytical stages, our laboratory has developed a comprehensive plan. These include; developing clear written procedures, augmenting training of health care professionals, monitoring quality indicators, enhancing communication between health care professionals and encouraging cooperation between departments.

\section{CONCLUSIONS}

The total testing procedure must be surveyed systematically so that in the present era with a high quality patient centered needs, there must be minimal mistakes.

\section{REFERENCES}

1. Plebani M, Sciacovelli L, Aita A, Padoan A, Chiozza ML. Quality indicators to detect pre-analytical errors in laboratory testing. Clin Chem Acta. 2014; 432:44-48.

2. Kohn LT, Corrigan JM, Donaldson MS. Institute of Medicine. Committee on Quality of Health Care in America. To err is human: building a safer health system. National Academic Press (US). 2000.[Pub Med]

3. Bankovic Radovanovic P, Kocijancic M. How well are pregnant women in Croatia informed about the oral glucose tolerance test?. Biochemia medica: Biochemia medica. 2015;25(2):230-6.[Full Text]

4. Plebani M, Sciacovelli L, Aita A, Padoan A, Chiozza ML. Harmonization of pre-analytical quality indicators. Biochemia Medica. 2014; 24(1):105-13. [Full Text]

5. American Diabetes Association. Diagnosis and Classification of Diabetes Mellitus. Diabetes Care. 2010; 33 (Supp1): S62-S69.

6. Burtis CA, Ashwood ER, Bruns DE. Tietz textbook of clinical chemistry and molecular diagnostics-e-book. Elsevier Health Sciences. 2012.[Full Text]

7. Shahangian S, Snyder SR. Laboratory medicine quality indicators: a review of the literature. Am J Clin Pathol. 2009;131(3):418-31. [Full Text]

8. Singla P, Parkash AA, Bhattacharjee J. Preanalytical error occurrence rate in clinical chemistry laboratory of a public hospital in India. Clin Lab. 2011;57(9-10):749-752.

9. Nutt L, Annalise EZ, Rajiv TE. Incomplete lab request forms: the extent and impact on critical results at a tertiary hospital in South Africa. Ann Clin Biochem. 2008;45:463466. doi: 10.1258/acb.2008.007252.

10. Koseoglu M, Hur A, Atay A, Cuhadar S. Effects of hemolysis interference on routine biochemistry parameters. Biochemia Medica. 2011;21(1):79-85.[Full Text]

11. Lippi G, Salvagno LG, Montagnana M, Brocco G, Guidi GC. Influence of hemolysis on routine clinical chemistry testing. Clin Chem Lab Med. 2006;44:311-6. [Full Text]

12. Najat D.Prevalence of Pre-Analytical Errors in Clinical Chemistry Diagnostic Labs in Sulaimani City of Iraqi Kurdistan. PLoS ONE 12(1): e0170211. [Full Text]

13. Zaini RG, Dahlawi HA, Siddiqi A. Identification of the Types and Frequencies of Pre-analytical Errors in the Clinical Biochemistry Laboratory: 1-year Study at Heraâ a General Hospital. Arch Med. 2016;8:4.[Full Text] 